Esquinas López, R. (2021). El audiolibro como TIC para la enseñanza y aprendizaje del léxico en el aula de E/LE

RILEX. Revista sobre investigaciones léxicas, 4/II, pp. 123-150.

\title{
EL AUDIOLIBRO COMO TIC PARA LA ENSEÑANZA Y APRENDIZAJE DEL LÉXICO EN EL AULA DE E/LE
}

\section{AUDIOBOOKS AS A ITC FOR THE TEACHING AND LEARNING OF LEXICON IN THE CLASS OF SFL}

\author{
Rosana Esquinas López \\ Universidad de Murcia \\ rosana.esquinasl@um.es
}

\begin{abstract}
RESUMEN
Los audiolibros son un recurso analizado, principalmente, en la enseñanza de inglés como lengua extranjera. Sin embargo, poco sabemos sobre su situación y uso en el ámbito de la enseñanza de español como lengua extranjera. En este trabajo nos centraremos especialmente en reflexionar sobre los beneficios del audiolibro como recurso para la enseñanza y aprendizaje de la competencia léxica revisando estudios previos con estudiantes de nivel intermedio, equivalente a los niveles B1-B2 del MCER y, concretamente, en el ámbito de la enseñanza de E/LE. Además, a través de un estudio cuantitativo en forma de encuesta dirigida a docentes de E/LE analizaremos su percepción sobre esta herramienta y sus posibles aplicaciones en el aula. Esta investigación evidencia el interés por los audiolibros por parte de los docentes, así como la falta de recursos editoriales especializados y propuestas educativas que faciliten su implementación en el aula de E/LE.
\end{abstract}

Palabras clave: españollengua extranjera; competencia léxica; léxico; audiolibros

\begin{abstract}
Audiobooks are a resource analyzed mainly in the field of teaching English as a foreign language. However, little is known about their situation and use in the field of teaching Spanish as a foreign language. In this paper we will focus especially on reflecting on the possibilities of the audiobook as a resource for the teaching and learning of lexical competence by reviewing previous studies with students of intermediate levels, equivalent to B1-B2 levels of the CEFR levels and, specifically, in the field of teaching Spanish as a foreign language. In addition, through a quantitative study in the form of a survey addressed to SFL teachers, we analyze their perception of this resource and its possible applications in the classroom. This research shows the interest in audiobooks on the part of teachers, as well as the lack of specialized editorial resources and educational proposals to facilitate their implementation in the classroom.
\end{abstract}

Keywords: Spanish as foreign language; lexical competence; lexicon; audiobooks 


\section{INTRODUCCIÓN ${ }^{1}$}

Los audiolibros están siendo uno de los principales productos digitales en auge a nivel mundial. Uno de los usos con mayor potencial y más denostado del audiolibro es el aprendizaje de una lengua extranjera; de hecho, la mayoría de las investigaciones en torno al mismo se centran en resaltar su potencial en cuanto a su aplicación para mejorar la destreza auditiva y oral. No obstante, poco sabemos sobre sus posibles usos y beneficios en cuanto a la adquisición del léxico, concretamente, en el aula de Español como Lengua Extranjera (E/LE, en lo sucesivo).

La aparición de internet y diferentes dispositivos tecnológicos como los móviles y tabletas, ha hecho posible que se expanda el uso de los audiolibros. En este sentido, varias investigaciones demuestran que el uso del audiolibro tiene beneficios muy notables a la hora de aprender lenguas extranjeras, sobre todo en el esfuerzo necesario para memorizar nuevas palabras y ampliar el conocimiento léxico (Chang, 2011). Por ello, ahondaremos en las posibilidades que podría tener el audiolibro en el aula de E/LE, un área de investigación poco explorada hasta la fecha. Sabemos que los audiolibros han demostrado ser útiles en el ámbito del aprendizaje de lenguas (Alcantud-Díaz y Gregori-Signes 2014, p. 111), pero desconocemos sus posibilidades de uso en el ámbito concreto de la didáctica del léxico en el aula de E/LE. No obstante, consideramos que los audiolibros entendidos como Tecnologías de la Información (TIC, en lo sucesivo) pueden ser un recurso útil y motivador para el aprendizaje y la enseñanza del léxico. A continuación, se presenta una reflexión teórica y práctica sobre la posible aplicación pedagógica de un recurso como los audiolibros y sus implicaciones. Por último, con el objetivo de dotar a esta investigación de datos reales y actualizados, expondremos los resultados de una investigación cuantitativa en forma de encuesta dirigida

\footnotetext{
${ }^{1}$ Esta investigación está basada en un trabajo de tesina fin de máster inédito dentro de la especialidad de Enseñanza del léxico en español como lengua extranjera.
} 
a docentes de E/LE cuyo propósito fue el de averiguar su percepción sobre este recurso y posibles aplicaciones en el aula.

\section{AUDIOLIBROS Y LÉXICO: ESTUUDIOS PREVIOS}

Los audiolibros han tenido su principal aplicación en hacer la literatura accesible para personas con problemas de visión que no podían acceder a la lectura en papel (Rubery, 2016). No obstante, este primer uso ha derivado en su aplicación en el aula de lenguas extranjeras, pues permite trabajar numerosas destrezas, tal y como indican diferentes investigaciones con estudiantes de nivel intermedio (Brown et al., 2008); Chang, 2011; Muñoz Coronado, 2012; Webb y Chang, 2015). Hoy en día, no existen audiolibros creados con fines didácticos, por lo que consideramos conveniente clasificarlos como un recurso didáctico y no como medio didáctico. Siguiendo la definición de Marquès Graells (2000) un medio didáctico es todo material elaborado con la intención de facilitar el proceso de enseñanza y aprendizaje, mientras que un recurso educativo es todo material que se emplee con fines didácticos pero que no ha sido creado con dicho fin.

Dada la escasez de estudios realizados en el ámbito específico de E/LE sobre la relación entre la enseñanza y aprendizaje del léxico a través de audiolibros, es necesario analizar los resultados de estudios que han analizado los beneficios de los audiolibros, pero en contextos de enseñanza de inglés como lengua extranjera. Por un lado, podemos afirmar gracias al estudio de Blum et al. (1995) que los aprendientes que en casa escucharon grabaciones de los mismos libros que leían incrementaron su capacidad de comprensión lectora y fueron capaces de leer textos más difíciles que al inicio del estudio. Los participantes de dicho estudio fueron cinco estudiantes internacionales con un uso limitado de la lengua inglesa e hicieron esta tarea durante 19 semanas. Estos resultados nos permiten afirmar que los audiolibros tendrían un impacto positivo en el aprendizaje del léxico en E/LE, sobre todo, si se acompañan del texto escrito. Si partimos de la base de que la competencia 
léxica está considerada como una subcompetencia que forma parte de la competencia comunicativa (Baralo, 2005, p. 27), podemos deducir que, si los participantes fueron capaces de leer y comprender textos de mayor dificultad, fue por el enriquecimiento léxico fruto de la actividad con audiolibros. Esta idea se consolida con investigaciones posteriores como la de Bergman (1999) cuyo estudio sobre el método Reading While Listening (RWL, en adelante) [leer mientras escuchamos] le lleva a afirmar que los audiolibros mejorar tanto la capacidad de reconocer palabras como la comprensión de estas. En la misma línea, le siguen investigaciones como la de Brown, Waring y Donkaewbua (2008) compararon tres formas de adquirir léxico nuevo: (1) a través de la lectura, (2) método RWL y (3) solo escucha. Los resultados que obtuvieron demuestran que los 35 estudiantes que participaron en el estudio adquirieron más léxico a través del método de RWL. Por lo tanto, cabe preguntarnos si lo idóneo sería aplicar este método también en el caso del aula de E/LE o como ejercicio fuera de la misma para la enseñanza y aprendizaje del léxico.

En este sentido, cobran especial importancia los resultados del estudio de Chang (2011) sobre la relación entre los audiolibros y el aprendizaje de léxico. No obstante, recordemos que este estudio se ha llevado a cabo en el contexto del aula de inglés como lengua extranjera. Esta investigación trata sobre el impacto de leer el mismo audiolibro que se escucha y su efecto en la destreza auditiva y la adquisición de vocabulario. El método de aprendizaje empleado vuelve a ser el método RWL. Dado que lo que analizamos en esta investigación es la enseñanza y aprendizaje del léxico a través del audiolibro, nos centraremos en los resultados de dicho aspecto. El estudio duró 26 semanas y contó con dos grupos, siendo un total de siete estudiantes los que formaron el grupo de RWL y el resto ejerció como grupo de control. Chang realizó test previos para averiguar de qué base de vocabulario partían los aprendientes y poder así comparar los resultados finales después de las semanas experimentales. Los materiales utilizados para el grupo de RWL fueron lecturas graduadas acompañadas de su correspondiente audiolibro, 
editados por editoriales especializadas en el ámbito de inglés como lengua extranjera como Cambridge y Oxford (Chang, 2011, p. 49). Los estudiantes de este grupo debían leer, al menos, un libro a la semana y podían utilizar los ordenadores del centro. Posteriormente, se reunían una vez a la semana con la investigadora para intercambiar opiniones sobre el libro escogido, ya que cada estudiante era libre de escoger uno u otro de la lista proporcionada. El resto de los estudiantes, los pertenecientes al grupo de control, tan solo escuchaban historias de elección propia, con una longitud de entre 300 y 500 palabras, y escuchaban una cada semana. Chang señala que el principal objetivo del estudio era comparar hasta qué punto se diferenciarían ambos grupos en términos de vocabulario y capacidad auditiva al final del experimento. En cuanto a las pruebas relacionadas con la comprensión auditiva Chang (2011, p. 52) indica que el grupo de RWL obtuvo mejores resultados de forma significativa respecto al grupo control en ambas tareas: pruebas de respuesta múltiple y el dictado. En este sentido, podemos afirmar que, los estudiantes que leyeron y escucharon el mismo audiolibro, fueron capaces de reconocer más palabras y aumentar su capacidad lectora y auditiva. De esta manera, los resultados de Chang corroboran su hipótesis sobre los beneficios de los audiolibros acompañados del texto en estudiantes de lenguas extranjeras. Igualmente, los resultados vuelven a ser mejores en términos de aprendizaje de vocabulario en el grupo de RWL respecto al grupo control. La autora estima que el grupo RWL aprendió 566 palabras y el grupo control 123, basándose en que las pruebas de vocabulario tienen una media de 33,3 palaras nuevas. En definitiva, una de las principales conclusiones de Chang es que todos los estudiantes del grupo RWL mejoraron sus destrezas. En términos concretos de vocabulario, tema principal de la presente investigación, Chang ha demostrado que la mejora en la adquisición de este es muy notable gracias al uso de audiolibros. Este aumento de vocabulario deriva en que los estudiantes del grupo RWL fueran capaces de leer textos más difíciles y largos al final del estudio y mejorar sus resultados en los dictados 
posteriores al estudio. Es decir, gracias al léxico aprendido a través del uso de audiolibros, los participantes del estudio de Chang (2011) fueron capaces de reconocer nuevas palabras y añadirlas a su lexicón mental. Sin embargo, la misma investigadora reconoce que los resultados de su estudio son el punto de partida para futuras investigaciones, dado que tuvo un número reducido de participantes. A este respecto, Chang (2011, p. 59) concluye que, el ejercicio de escuchar textos mientras se lee, demostró un claro aumento en la adquisición de vocabulario, mejorías en la comprensión tanto oral como escrita e interés en consumir más literatura. Consideramos que sin la mejoría en la adquisición de nuevo léxico estos resultados no habrían sido posibles; por lo que, una vez más, los audiolibros se presentan como un recurso con potencial demostrable en la enseñanza y aprendizaje de léxico en el aula de E/LE. Igualmente, si la competencia léxica está considerada como una subcompetencia que forma parte de la competencia comunicativa, entendemos que la mejoría del léxico que deriva en mejores resultados en tareas como el dictado. Por ello, podemos coincidir con los apuntes de Dirven y Oakeshoot-Taylor (1984) quien indica que los dictados son tareas que requieren tanto conocimiento lingüístico (fonética, ortografía, morfología, etc.) como reconocimiento léxico y análisis sintáctico. Por lo tanto, podemos afirmar que el modelo de lectura que proponen los audiolibros permite mejorar el léxico del aprendiente.

De igual forma, dado que la mayoría de las plataformas que ofrecen audiolibros permiten regular la velocidad de lectura, el audiolibro permitiría solucionar uno de los problemas principales que experimentan los estudiantes de lenguas extranjeras en ejercicios de destreza auditiva como es el de no poder adaptarse a la velocidad del audio. Investigaciones como la de Chang y Read (2006) afirman que entre las dificultades más señaladas por los aprendientes durante los ejercicios de destreza auditiva es un ritmo muy rápido, incapacidad de pensar suficientemente rápido para entender el input del ejercicio y no ser capaz de relacionar lo escuchado con lo escrito. El estudio 
de Chang (2011, p. 46), vuelve a subrayar el aprendizaje autónomo que permite el audiolibro, pues se puede usar fuera del aula y ser un complemento a las horas limitadas de la clase formal, motivo que podemos suponer es el principal escoyo con el que se encuentran los docentes a la hora de diseñar actividades teniendo en cuenta su uso.

Por otro lado, uno de los estudios consultados ha sido el llevado a cabo por Alcantud-Díaz y Gregori-Signes (2014) en el que examinan cómo los audiolibros pueden mejorar la fluidez e inculcar habilidades literarias en el aula de inglés como lengua extranjera en primaria. Si bien es cierto que este estudio vuelve a estar enfocado a la enseñanza de la lengua inglesa, podríamos aplicar la mayoría de las conclusiones al contexto del aula de E/LE. Las autoras consideran que los audiolibros pueden contribuir a la mejora de una de las destrezas recogidas en el Marco Común Europeo de Referencia (MCER, en lo sucesivo) como es la capacidad auditiva. Los audiolibros, en este sentido, afirman que pueden servir como modelo al ser muestra de diferentes dialectos, ritmos, pausas y voces (Alcantud-Díaz y Gregori-Signes, 2014, p. 14). Por lo tanto, podríamos considerar que mejora la competencia léxica del aprendiente, pues ambas competencias están relacionadas. Igualmente, es estudio subraya otros aspectos positivos del uso de audiolibros en el aula de lengua extranjera, como la posibilidad de aislamiento y concentración que se consigue con el uso de auriculares. Asimismo, señalan la importancia que podrían tener los audiolibros para los aprendientes con necesidades especiales que no pueden disfrutar del método de lectura tradicional, aspecto que juega a favor del audiolibro (cf. Wittingham et al., 2013).

\subsection{Audiolibros y LÉXICO EN E/LE: ESTUDiOS PREVIOS}

La aparición del audiolibro en los materiales de E/LE es escasa o prácticamente inexistente, por este motivo escasean también los estudios dedicados a audiolibros en el aula de E/LE y, más concretamente, sobre su relación con 
la competencia léxica. Sin embargo, podemos examinar estudios como el de Muñoz Coronado (2012), García Giménez (2009) y Vallorani (2011).

Por un lado, Muñoz Coronado (2012, p. 83) define los audiolibros como "recursos tecnológicos en formato audio alternativo a la lectura convencional, de gran utilidad para aprender y enseñar idiomas". La misma investigación también destaca que los audiolibros como recurso en este contexto concreto tienen un doble objetivo, siendo (1) una herramienta que ayuda a diversificar las tareas del docente como la elaboración de contenidos; y, (2) un recurso que permiten el autoaprendizaje, donde la utilización por parte del alumno mejora sus destrezas. A través del estudio de Muñoz Coronado (2012, p. 95) podemos reflexionar sobre los beneficios que pueden aportar los audiolibros en la enseñanza de E/LE, siendo estos: (1) diversificación del abanico oral, en tanto que ofrecerán diferentes acentos, pronunciaciones, vocabulario y expresiones idiomáticas; (2) motivación del aprendiente, pues se estimula su autonomía y se le otorgan las riendas de su propio aprendizaje; (3) entrenamiento eficiente de destrezas orales y auditivas, facilitando la comprensión de lo que escuchan; (4) acercamiento de la literatura y cultura española e hispanoamericana; (5) introducción de temas, autores o situaciones con contenidos auténticos, novedosos y llamativos en clase; (6) inclusión de estudiantes con dificultades como discapacidad visual o invidentes; (7) diversificación de patrones de entonación, de discurso, registros y estilos de conversación; (8) libertad de movimiento al ser un formato que permite la movilidad y la simultaneidad con otras tareas.

Por otro lado, trabajos como el de García Giménez (2008, p. 9) indican que "la oralidad es un elemento fundamental para obtener el éxito en el proceso de adquisición de una lengua extranjera, pues las destrezas lingüísticas pueden aprenderse mejor si los elementos de la lengua meta se presentan de forma oral antes de verse de forma escrita". Es decir, partir de lo oral para afianzar el aprendizaje antes de trabajar los contenidos de forma escrita. Asimismo, su investigación dirigida a aprendientes de E/LE atestigua que los mismos 
reclaman más práctica auditiva por considerarla fundamental para la fijación de contenidos léxicos (García Giménez, 2008, p. 48). Por ello, el audiolibro podría contribuir a este tipo de prácticas para la enseñanza y aprendizaje de la competencia léxica. Por su parte, Vallorani (2011, p. 65) afirma que "la escucha de un libro con el texto delante reduce hasta el 30\% el tiempo necesario para memorizar nuevas palabras", tanto es así, que con una lectura silenciosa del texto considera que se crea una verdadera amplificación del aprendizaje tanto de lenguas extranjeras como de la propia lengua materna".

En definitiva, los beneficios de los audiolibros son múltiples y merecen un análisis exhaustivo en el aula de E/LE; pues este recurso puede suponer un acercamiento a la cultura meta, a incentivar la lectura y a trabajar múltiples competencias contempladas en el MCER, recurso vertebrador de la enseñanza de lenguas extranjeras a nivel europeo.

\section{EST'UDIO: JUSTIFICACIÓN Y PREGUNTAS DE INVESTIGACIÓN}

Dada la escasez de estudios empíricos sobre el uso de audiolibros en E/LE, a través del presente estudio se busca dotar de datos reales a esta investigación alejándonos de lo teórico para analizar la situación real desde el punto de vista práctico. Por ello, con el objetivo de examinar el potencial de los audiolibros, opinión y posibles experiencias de los docentes con dicho recurso nos planteamos las siguientes preguntas de investigación:

(1) ¿Hasta qué punto son los audiolibros un recurso desconocido, interesante y poco empleado en el aula de E/LE?

(2) ¿Se relaciona estrechamente el uso del audiolibro con la enseñanza y aprendizaje de la competencia léxica?

(3) ¿Los docentes disponen y conocen maneras de poner en marcha propuestas educativas que incluyan el audiolibro?

\subsection{MÉTODO}

La encuesta se diseñó utilizando la herramienta de Google Forms y estaba dirigida a docentes de E/LE de todo el mundo, motivo por el cual se distribuyó a través de internet y redes sociales como Twitter y Facebook. 
La encuesta constaba de 16 preguntas obligatorias y una última opcional, pues se trataba de un espacio en el que los participantes pudieran añadir cualquier observación o comentario que considerasen de interés para el propósito del estudio. A su vez, la encuesta estaba dividido en tres secciones. La primera sección tenía el objetivo de crear un perfil demográfico sobre los participantes. Por lo tanto, se les preguntó por su grupo de edad y país de residencia. En la segunda sección, se recogieron datos sobre el perfil profesional en cuanto a formación máxima alcanzada y años de experiencia.

La tercera sección recoge preguntas en las que se les pedía a los docentes que indicaran si alguna vez habían hecho una actividad con audiolibros o se habían encontrado con algún manual o propuesta educativa en la que se indicase su uso. Asimismo, se les preguntó si se consideraba que audiolibro debería incluirse en los manuales especializados como recurso TIC. En la cuarta sección se exploró qué competencias se relaciona el uso del audiolibro y estas debían responderse utilizando una escala de Likert del 1 al 5, siendo 1 Nada útil y 5 Extremadamente útil. Las competencias por las que se preguntaron fueron: (1) comprensión auditiva; (2) comprensión lectora; (3) comprensión e interacción oral; (4) competencia léxica y (5) competencia sociolingüística. Además, dado el enfoque en la enseñanza del léxico de esta investigación, se propuso una pregunta sobre qué aspectos de la competencia léxica consideraban los participantes que podrían trabajarse con el audiolibro, siendo: marcadores del discurso; español coloquial y argot; vocabulario activo y pasivo u otro. Se seleccionaron estos aspectos con el objetivo de acotar en cierto modo el ámbito de la investigación.

Dado el escaso número de investigaciones sobre el audiolibro en E/LE, la quinta sección tiene el objetivo de recoger la opinión de los docentes sobre dicho recurso. Por lo tanto, se pidió a los participantes que seleccionasen la opción que más se ajustase a su opinión sobre el audiolibro. Igualmente, se 
dejó una opción de respuesta abierta para dar la posibilidad de expresar una opinión diferente a las propuestas. Las opciones fueron las siguientes:

(1) Alguna vez me he planteado su uso, pero me siento incapaz al no disponer de

(2) suficiente información sobre cómo emplearlo en el aula.

(3) Alguna vez me he planteado su uso, pero como tarea extraescolar.

(4) Alguna vez me he planteado su uso, pero acompañando la escucha con el texto

(5) delante.

(6) Nunca me he planteado su uso.

(7) Otro

Igualmente, se les pidió que, de una lista de posibles actividades con el audiolibro basadas en la propuesta de Muñoz Coronado (2012), seleccionasen las que considerasen más acertadas. Se volvió a dejar la opción de respuesta abierta para aquellos participantes que quisieran indicar otro tipo de actividad. Las propuestas de trabajo fueron las siguientes:

(1) Trabajar en clase con texto y audio: ejercicios de rellenar huecos, verdadero/ falso, preguntas y respuestas, etc.

(2) Trabajar el español en el mundo: diferentes construcciones, expresiones y palabras.

(3) Trabajar el contexto del autor y el texto: hipótesis, significado, anécdotas, adivinar el título, relacionar el léxico, etc.

(4) Actividades extraescolares: exposiciones, presentaciones, taller de lectura, grabación de lectura de poemas, edición de audiolibros, etc.

(5) Otro.

\subsection{Perfil de la muestra}

Los participantes en este estudio fueron un total de 80 docentes de E/LE de los cuales el 29\% ejercen su profesión en España, seguido del porcentaje más alto (10\%) residentes en Italia. No obstante, dado que la encuesta se distribuyó por internet, este llegó a docentes de diferentes partes del mundo como Alemania, Brasil, Estados Unidos y Turquía. A pesar de la anonimidad de la encuesta, con el objetivo de obtener datos sobre el perfil de docente, se lanzaron preguntas para averiguar sus años de experiencia y formación máxima alcanzada. En el caso de nuestro estudio, el 53\% de los participantes tenían más de siete años de experiencia, por lo que podemos afirmar que 
los resultados obtenidos son fiables, pues en su mayoría proceden de docentes con una amplia experiencia docente. En cuanto a la formación máxima alcanzada, la mayoría de los encuestados (30\%) ha cursado una Licenciatura o Grado en Filología, Traducción o Lenguas Modernas, seguido de un 23\% que ha cursado un Máster en Enseñanza de Español como Lengua Extranjera. De nuevo, podemos afirmar que los encuestados cuentan con una opinión fundamentada en su formación y experiencia en el contexto concreto de la enseñanza de E/LE.

\subsection{RESULTADOS Y ANÁLISIS}

Los resultados estadísticos arrojan luz sobre cómo perciben los docentes de E/LE el audiolibro, para qué competencias podría ser más útil y el tipo de actividades idóneas para emplear su uso.

\section{Preguntas n..$^{\circ}$, 5 y 6.}

(1) Los audiolibros tienen uno de sus principales usos en el aprendizaje de lenguas extranjeras. ¿Alguna vez has hecho una actividad con audiolibros?

(2) ¿Alguna vez te has encontrado con algún manual o propuesta de actividades que indicase el audiolibro como parte de la actividad?

(3) ¿Consideras que sería interesante que los manuales especializados tuvieran en cuenta el audiolibro como recurso TIC?

El objetivo de estas preguntas era conocer si los docentes habían realizado alguna actividad con audiolibros o si se habían encontrado con algún manual o propuesta que indicase el audiolibro como parte de la actividad. Igualmente, nos interesaba averiguar si consideraban que los manuales especializados debían tener en cuenta al audiolibro como recurso TIC. Ante estas preguntas, el 70\% de los participantes indica que nunca han realizado una actividad con audiolibros. Sin embargo, podemos relacionar de forma directa que esto se debe a que el $77,5 \%$ indica que nunca se ha encontrado con un manual o actividad que propusiera su uso. A pesar de la falta de actividades o manuales especializados que propongan actividades con audiolibros, una amplia mayoría representada por el $95 \%$ de los encuestados consideran 
que sería interesante que los manuales especializados tuvieran en cuenta el audiolibro como recurso TIC.

Los resultados de estas preguntas nos permiten contestar dos de las tres preguntas de nuestra investigación. El audiolibro es, en efecto, un recurso desconocido y poco empleado en el aula de E/LE, pero de gran interés (pregunta de investigación $n^{\circ} 1$ ). Además, este desconocimiento general deriva en que los docentes ignoren maneras de poner en marcha propuestas didácticas que incluyan audiolibros (pregunta de investigación $n^{\circ} 3$ ).

Pregunta n..$^{\circ}$. Selecciona las competencias y destrezas que consideran podría trabajarse con el audiolibro.

El objetivo de esta pregunta era averiguar qué competencias relacionadas con la enseñanza y aprendizaje de español consideraban los docentes que podrían trabajarse con el audiolibro. En la Figura 1 podemos observar que la mayoría consideran que la comprensión auditiva, seguida de la competencia léxica son las principales competencias en las que el audiolibro podría incluirse como recurso.

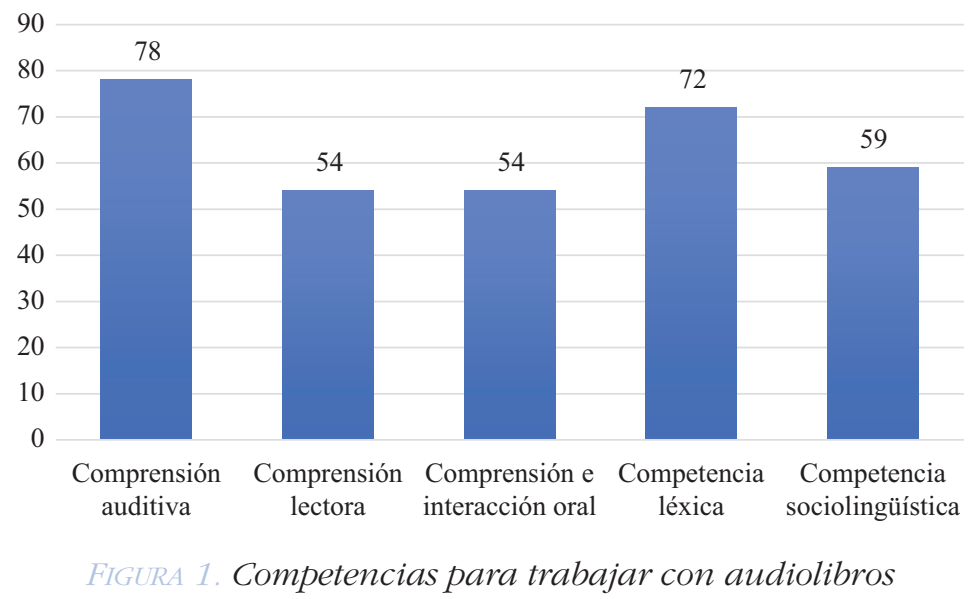

Estos porcentajes nos permiten afirmar que la competencia léxica se percibe como una de las principales competencias a desarrollar a través del audiolibro. Como es lógico, dado el componente sonoro del audiolibro, se relaciona 
estrechamente su uso con el desarrollo de la comprensión auditiva, pues el 97\% de los participantes señalaron esta opción frente al 90\% que escogió la competencia léxica, ocupando el segundo puesto. No obstante, estos resultados podrían relacionarse con el estudio desarrollado por Chang (2011) que hemos analizado previamente. Sin embargo, llama nuestra atención que sea la competencia sociolingüística la tercera en mayores porcentajes $(73,8 \%)$, pues no habíamos considerado esta opción al inicio de nuestra investigación. En este sentido, podríamos abrir una nueva vía de aplicación de los audiolibros como recurso para el desarrollo de dicha competencia.

Estos resultados nos permiten contestar la pregunta de investigación n. ${ }^{\circ}$ 2 y podemos señalar que el audiolibro no se relaciona de forma directa con la competencia léxica, sino que comparte este espacio con la comprensión auditiva. No obstante, consideramos que son competencias que se alimentan la una de la otra, pues sin el léxico no podemos comunicarnos ni descifrar ningún mensaje, por lo que la comprensión auditiva contribuye a la mejora de la competencia léxica y viceversa.

Preguntas $.^{\circ} 8,9,10,11$ y 12. Estas preguntas se formularon para que fueran contestadas usando la escala de Likert, es decir, con escalas del 1 al 5. El objetivo de estas era averiguar el grado de utilidad de los audiolibros dependiendo de la competencia o destreza a trabajar. Las diferentes competencias propuestas fueron: (8) la comprensión auditiva; (9) comprensión lectora; (10) comprensión e interacción oral; (11) competencia léxica; y, por último, (12) la competencia sociolingüística.

La escala Likert iba desde el 1, cuyo valor era para nada útil hasta el 5 con el valor de extremadamente útil. Los resultados de estas preguntas nos permiten responder nuestra segunda pregunta de investigación que era averiguar con qué competencias y destrezas se relaciona el audiolibro según los docentes. De nuevo, tal y como podemos observar en las siguientes figuras, son la comprensión auditiva y la competencia léxica las que obtienen los porcentajes más altos. 
El porcentaje es algo menor respecto a la pregunta anterior, en la que el 97\% de los encuestados se decantaban por la competencia auditiva como la idónea para utilizar los audiolibros. Sin embargo, sigue siendo la opción principal para usar audiolibros, tal y como podemos observar en la Figura 2. Igualmente, como podemos observar en la Figura 3, la competencia léxica obtiene resultados altos con un 37,5\%. Es decir, 30 de los 80 encuestados perciben que los audiolibros podrían ser extremadamente útiles para trabajar la competencia léxica. Mientras que un 32,5\% la percibe como muy útil.
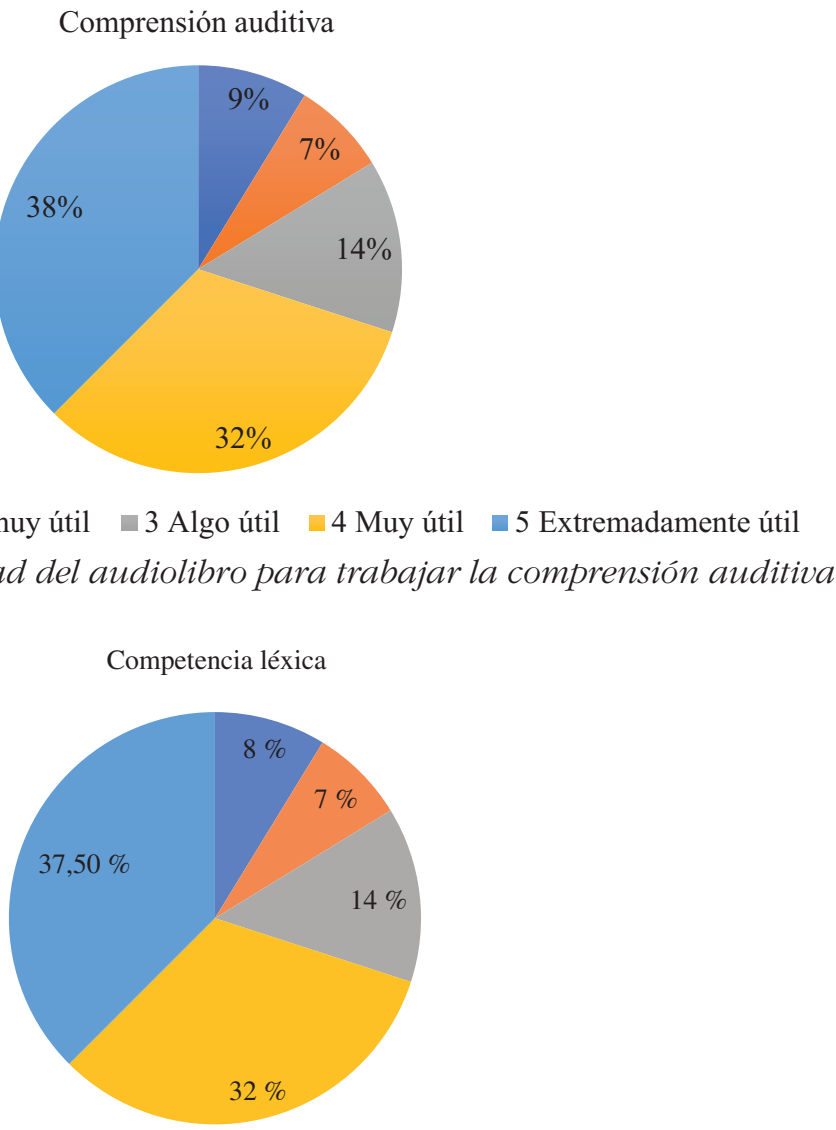

• Para nada útil $₫$ No muy útil $₫$ Algo útil $\llbracket$ Muy útil $₫$ Extremadamente útil

FIGURA 3. Grado de utilidad del audiolibro para trabajar la competencia léxica

Las opciones con porcentajes más bajos fueron la comprensión lectora, la comprensión e interacción oral y la competencia sociolingüística. Tal y como se puede observar en las Figura 3, la competencia sociolingüística se percibe 
como la que menos podría beneficiarse del uso del audiolibro. Esto último contrasta con los resultados de la pregunta anterior en la que la competencia sociolingüística ocupa el tercer lugar con un porcentaje elevado $(73,8 \%)$ de participantes que la escogieron como una de las competencias que podrían trabajarse con el audiolibro, como ilustra la Figura 4.

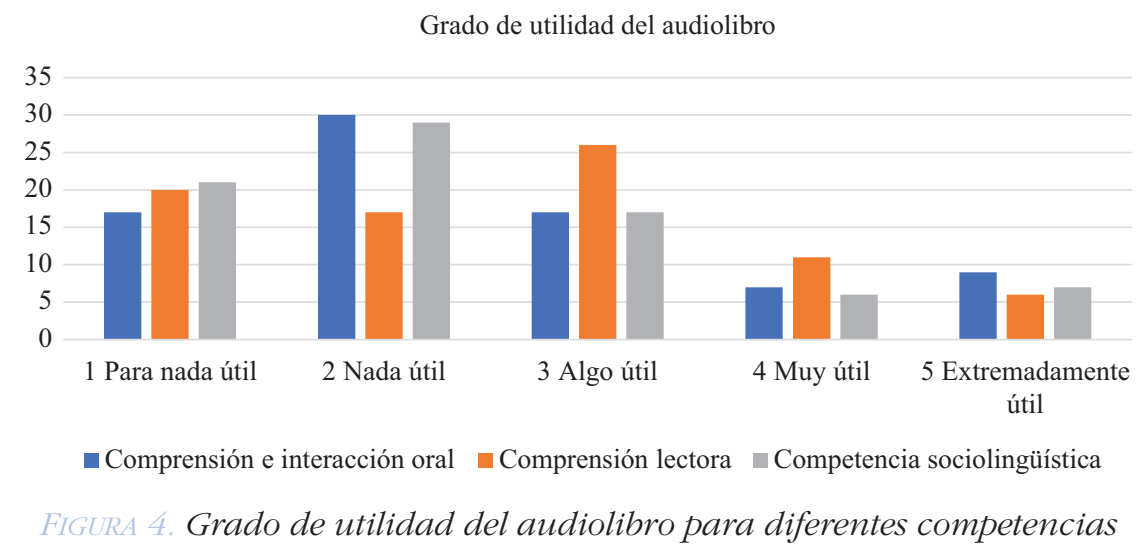

Pregunta n..$^{\circ}$ 13. Selecciona los aspectos de la competencia léxica que consideras podrían trabajarse con el audiolibro.

Esta pregunta tiene el objetivo de analizar para qué aspectos del léxico concretos podría ser más útil este recurso. Así, se le dieron tres opciones a los encuestados, siendo (1) marcadores del discurso; (2) español coloquial y argot y (3) vocabulario activo y pasivo. Asimismo, se dejó una opción de respuesta abierta para que el docente que lo considerase necesario indicase otro aspecto de la competencia léxica que podría trabajarse con los audiolibros.

Como refleja la Figura 5, la mayoría de los docentes encuestados consideran que el vocabulario activo y pasivo sería el aspecto de la competencia léxica que mejor podría trabajarse con los audiolibros. Sin embargo, dado que los encuestados podían seleccionar tantas opciones como quisieran, vemos que el porcentaje de las tres es bastante alto. El porcentaje menor lo obtienen los marcadores del discurso, a pesar de tener un $81,3 \%$ de resultados. 


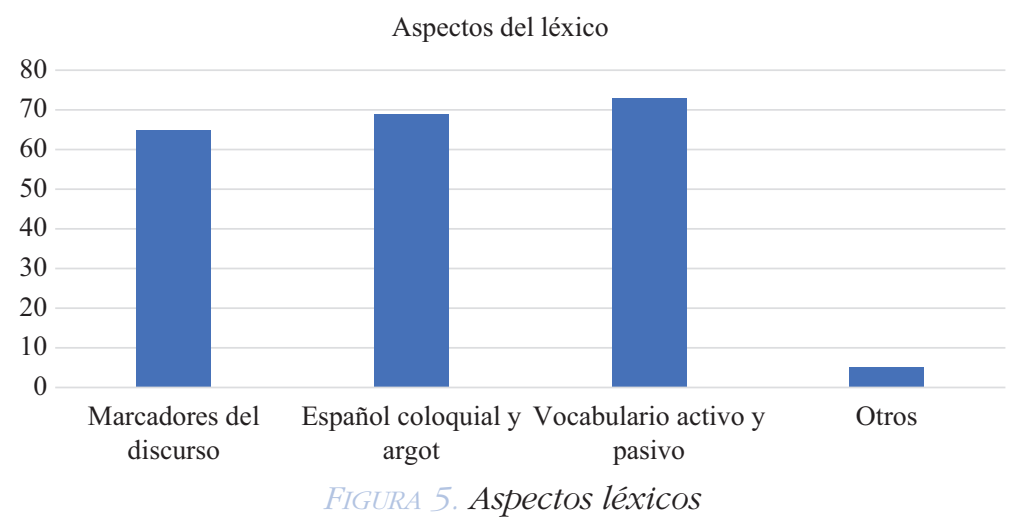

Tan solo cinco de los 80 encuestados aportaron otras opciones que, a su juicio, podrían trabajarse con audiolibros en relación con la enseñanza y aprendizaje del léxico en E/LE. Las propuestas fueron:

(1) Formalidades lingüísticas

(2) Cultura, pragmática

(3) Situaciones de la vida real

(4) Fraseología

(5) Competencia intercultural

Pregunta n..$^{\circ}$ 14. Selecciona la opción que más se ajuste a tu opinión sobre el audiolibro.

El propósito de esta pregunta era el de conocer la opinión sobre el audiolibro como recurso en el aula. De nuevo, se proponían cuatro opciones, pero se le daba la posibilidad al encuestado de indicar otra opción.

Observemos los resultados ilustrados en la Figura 6. El porcentaje más alto, representando al 26\% de los docentes, lo obtuvo la opción de Alguna vez me be planteado su uso, pero con el texto delante. En este sentido, podríamos reforzar esta opción de uso como muy positiva, dados los resultados de estudios analizados previamente como el de Chang (2011) donde se observan los beneficios del método RWL, es decir, de escuchar y leer el mismo texto. Cabe señalar que la segunda opción más elegida fue la de Nunca me he planteado su uso, representando al $24 \%$ de los encuestados. A pesar del contraste entre las dos opciones escogidas, muchos participantes expresaron opiniones interesantes en el apartado de respuesta abierta. Entre otros, los comentarios fueron: 
(1) Tarea extraescolar y con el texto delante

(2) Me he planteado muchas veces su uso, pero no le he dedicado el tiempo para hacerlo

(3) Me he planteado su uso, pero me ha resultado complicado en un contexto de enseñanza en línea

(4) Utilizo muchos audios, pero no libros completos. Empleo audios de cuentos, de poemas, de canciones y de conversaciones. Cuando digo que no empleo audiolibros ni clase es porque no lo hago con libros completos.

(5) Los uso para trabajar todas las competencias. Lo que hay es que saber trabajar con ellos.

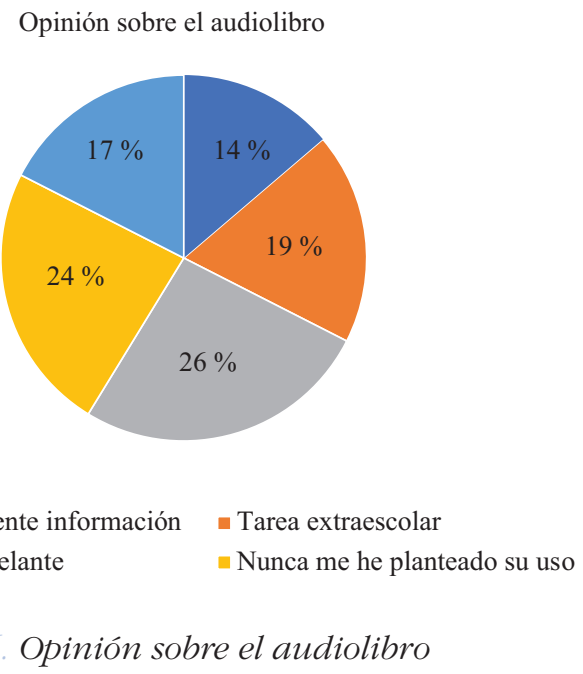

Pregunta n. ${ }^{\circ}$ 15. Dada la escasez de propuestas en actividades con audiolibros, consideramos interesante averiguar qué tipo de actividades se percibían como idóneas. Para ello, nos basamos en la propuesta del trabajo de Muñoz Coronado (2012, p.96) en el que se proponen cuatro actividades para implementar el audiolibro en el aula de E/LE. De nuevo, se volvió a dejar una opción de respuesta abierta para quien considerase oportuno añadir otras opciones.

Los resultados muestran que la opción más destacada es Trabajar el contexto del autor y el texto: hipótesis, significado, anécdotas, adivinar el título, relacionar el léxico, etc. Esta opción fue la escogida por 55 de los 80 participantes en el estudio, representado de esta manera al 68,8\%. En segundo lugar, con un porcentaje algo menor (52\%), se sitúa la opción Actividades extraescolares: exposiciones, taller de lectura, grabación de lectura de poemas, 
edición de audiolibros, etc. En tercera posición, encontramos la opción de Trabajar el español en el mundo: diferentes construcciones, expresiones y palabras. El 48\% de los docentes prefieren esta opción como actividad para trabajar audiolibros, consideramos que es una actividad relacionada con la enseñanza y aprendizaje del léxico, por lo que resulta positivo para el interés de la presente investigación. A pesar de haber expuesto anteriormente los beneficios del método RWL, la opción con menor porcentaje, aunque sea algo elevado, ha sido la actividad de Trabajar en clase con texto y audio: ejercicios de rellenar buecos, verdadero/falso, preguntas y respuestas, etc. En el caso de esta opción, el porcentaje es de 46,3\%, podemos suponer que esto es resultado de falta de tiempo para realizar este tipo de actividades en una sesión. Por último, tan solo tres de los 80 participantes indicaron actividades alternativas a las propuestas u opiniones, siendo:

(1) Input para la pronunciación

(2) A los alumnos les gusta leer y escuchar el mismo texto

(3) Ninguna me convence, veo la lectura extensiva como una tarea personal

Pregunta n. ${ }^{0}$ 16. A continuación, se orece una lista de plataformas generales y especializadas en E/LE que ofrecen audiolibros. Selecciona las que conozcas.

Por último, nos interesaba averiguar qué plataformas generales y especializadas en la enseñanza de E/LE conocían los docentes encuestados. En la lista se podían seleccionar tantas opciones como se conociesen. La Figura 7 refleja los resultados de este apartado de preguntas. No obstante, se volvió a dejar una opción de respuesta abierta, pues consideramos que sería interesante conocer otros recursos diferentes a los listados en la pregunta. Los resultados indican que el recurso más conocido fue, como era de esperar, la Biblioteca Electrónica del Instituto Cervantes (66,3\%), seguido de los recursos especializados ofrecidos por Habla con Eñe: Español Lengua Extranjera (31,3\%) y, en tercer lugar, la plataforma de uso general Storytel (28,7\%). Consideramos positivo que las opciones más señaladas sean recursos especializados, pues son recursos diseñados con el objetivo de implementarse en el aula de E/LE, 
lo cual facilitaría el trabajo de los docentes. No obstante, es llamativo que 19 de los 80 participantes, es decir, el 23,8\% indicaron no conocer ninguno de los recursos indicados. Esto último también nos lleva a pensar que existe un desconocimiento generalizado sobre dónde encontrar audiolibros que puedan usarse en el aula.

Actividades con audiolibros

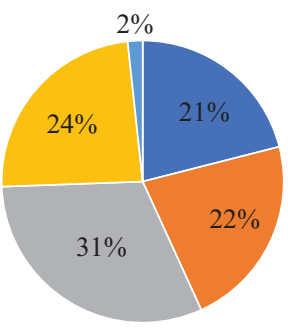

$$
\begin{aligned}
& \text { - Texto y audio } \\
& \text { - Contexto del autor y el texto } \quad \text { El español en el mundo } \\
& \text { - Otras } \\
& \text { FIGURA 7. Actividades con audiolibros }
\end{aligned}
$$

La última parte de la encuesta es un espacio para que el docente que lo considerase oportuno dejara algún comentario u observación sobre la investigación. Finalmente, 20 de los 80 participantes expresaron su opinión o valoración sobre nuestro estudio. La mayoría coinciden en señalar que el audiolibro en E/LE es un tema necesario sobre el que investigar, pero sobre el que escasean los recursos en el aula. Nos gustaría plasmar algunas de las opiniones más interesantes reflejadas en este espacio de la encuesta. Dado que algunas coinciden, las agruparemos en diferentes categorías, siendo el Grupo A aquel que expresa que el audiolibro es un recurso interesante, pero desconocido.

(1) Creo que es muy importante trabajar los audiolibros en el aula de ELE, aunque aún no he encontrado la manera perfecta de aplicarlo.

(2) Creo que es un aspecto muy interesante y de gran beneficio para los alumnos, aunque no se encuentra muy extendido.

(3) La verdad no conozco audiolibros adaptados por nivel para la clase de ELE, pero me parece muy interesante explorar esta idea como recurso.

(4) Sería realmente interesante tener propuestas didácticas para utilizar audiolibros en la clase de ELE. 
(5) Me parece un tema interesantísimo y que, desde luego, muchos profesores de ELE deberían incluir en sus clases si todavía no lo han hecho.

(6) Con los cambios que experimentamos en el mundo hoy día pienso que hay muchas más opciones de trabajar esta opción que investigas. Los estudiantes van a tener que responsabilizarse más de su propio aprendizaje y ampliar el foco. Escuchar audio libros en la lengua extranjera que estudien será atractivo, quizás ya lo hacen en su propia lengua en los largos desplazamientos en transporte público que hay en nuestras grandes ciudades.

Por su parte, encontramos un segundo grupo al que denominaremos Grupo B que expresan que el audiolibro es un recurso útil y utilizado.

(1) Me parece un tema interesantísimo y que, desde luego, muchos profesores de E/LE deberían incluir en sus clases si todavía no lo han hecho.

(2) Son de muchísima utilidad en los niveles más bajos. Este año he utilizado los de Lola Lago con mis alumnos de A2 y les han encantado.

\section{DISCUSIÓN}

Los resultados obtenidos a partir del análisis de los datos nos permiten afirmar que los audiolibros son un recurso altamente desconocido, interesante y poco empleado en el aula de E/LE, lo que nos permite responder la primera pregunta de investigación. Tal y como hemos podido ver en el apartado anterior, la mayoría de encuestados consideran que el método ideal sería trabajar con el audiolibro y el texto delante, pero es llamativo que un porcentaje relativamente elevado (24\%) indiquen que nunca se han planteado su uso y que un 14\% explicite que no disponen de suficiente información. Esto nos puede llevar a volver a la reflexión sobre la importancia de la actualización sobre las TIC por parte de los docentes, si consideramos el audiolibro como tal. Sin esta actualización, no es sencillo que se implemente su uso (Cruz Piñol, 2015). Como consecuencia, es necesario que, tanto creadores de materiales especializados como docentes de E/LE, exploren estas nuevas herramientas que surgen con los avances tecnológicos y que pueden contribuir a la enseñanza y aprendizaje de la competencia léxica tanto dentro como fuera del aula. Si bien es cierto que uno de los principales aspectos que juega en contra de los audiolibros es su duración, desconocemos si es uno de los 
principales motivos por el que la opción sobre su uso como tarea extraescolar tenga un porcentaje algo elevado (19\%). Asimismo, si consideramos el audiolibro como TIC, es necesario tener en cuenta que se presentan más bien como un complemento y no aseguran el aprendizaje si el docente pierde de vista aspectos como la tarea final, objetivos comunicativos, secuenciación de la actividad, así como la gestión de grupos y medios que se emplearán para la evaluación (Hernández Mercedes, 2012, p. 64). Sin embargo, trabajos como los de Herrera y Conejo (2009) ven en documentos organizadores como el MCER, un aspecto a favor en el uso de las TIC, pues afirman que, el hecho de que el MCER abogue por un enfoque orientado a la acción en la enseñanza de lenguas extranjeras, favorece el uso de las TIC en tanto que los hablantes son vistos como agentes sociales que usan la lengua para actuar y resolver tareas de la vida cotidiana. Por ello, los audiolibros podrían ser beneficiosos siempre que el docente sepa cómo trabajar con ellos y las actividades tengan un objetivo de consecución claro y delimitado. Es decir, que el audiolibro atienda a la necesidad del grupo y los objetivos de la actividad.

Al reflexionar sobre los resultados de las preguntas dirigidas a averiguar si el audiolibro se relaciona estrechamente con la enseñanza y aprendizaje de la competencia léxica, hemos podido constatar que se percibe como útil para dos componentes de la lengua: la comprensión auditiva y la competencia léxica. Lo cual nos permite contestar parcialmente a nuestra segunda pregunta de investigación. No obstante, esto no se considera algo negativo, pues tal y como indica Higueras (2017, p. 14) "la enseñanza del léxico no se concibe como un fin en sí mismo, sino supeditado a la mejora de la competencia comunicativa del alumno". Por ello, la competencia léxica influye en el resto de las competencias y esto provoca que los participantes la relacionen estrechamente con otras, como podría ser el caso de la comprensión auditiva. Si bien es probable que estos resultados tan similares nos puedan llevar a pensar de nuevo en la escasa información sobre las aplicaciones idóneas del audiolibro en el aula de E/LE. Por otro lado, es llamativo que el audiolibro 
haya tenido un alto porcentaje en cuanto al grado de utilidad para trabajar los tres aspectos del léxico propuestos, como son: (1) vocabulario activo y pasivo; (2) español coloquial y argot; y (3) marcadores del discurso. En primer lugar, el vocabulario activo y pasivo obtiene el mayor porcentaje y es positivo si nos apoyamos en las palabras de Chiriboga (1993, p. 28) quien indica que "en la enseñanza de vocabulario activo se debe emplear más tiempo, dando ejemplos y haciendo preguntas, para que los alumnos vean cómo se usa realmente esa palabra". El audiolibro permite que el aprendiente pueda retroceder y avanzar a su ritmo para asimilar el uso de estas nuevas palabras en un contexto concreto. Así, el audiolibro favorece el aprendizaje de vocabulario pasivo en tanto que puede adquirirse al deducir su significado por el contexto. Así, el aprendizaje la adquisición de ambos a través de audiolibros contribuye a aumentar el lexicón mental del aprendiente. En cuanto al español coloquial y argot, este recurso podría contribuir a suplir la falta de materiales didácticos experiencia necesaria para abordar la enseñanza de lenguaje coloquial y argot que indica Garrido Rodríguez (2003, p. 330) y que lleva a los docentes a "optar por la opción más rápida, sencilla pero menos eficaz: recurrir a listados de frases hechas, refranes y lenguaje argótico”. Este apunte coincide con los resultados de Chang (2011, p. 44) pues comprobó que con los audiolibros los aprendientes descubren usos contextualizados de lenguaje coloquial y argot que rara vez se encuentra en manuales escritos. Consideramos pues que el audiolibro en el aula de E/LE podría contribuir a trabajar la enseñanza y el aprendizaje del español coloquial, atendiendo a la enseñanza a nivel fonético, morfosintáctico, léxico-semántico y pragmático a través de una muestra real de lengua y donde podría haber variedad de hablantes. Por último, los marcadores del discurso también se perciben como un aspecto léxico que podría beneficiarse en gran medida del audiolibro. En este sentido, seguimos la propuesta de Nogueira da Silva (2009) en la que se propone la enseñanza de marcadores del discurso del español en relación con los géneros y secuencias textuales. Igualmente, Nogueira da Silva (2010) 
indica que el hecho de que algunos profesores no suelan saber interpretar el carácter procedimental, es decir "su ausencia de significado denotativo o designativo, lo que la diferencia del significado léxico estructurado de otras palabras" (2010, p. 4), suele derivar en no saber cómo enseñarlos. Así, consideramos que el audiolibro podría atender a esa necesidad de enseñar los marcadores dentro del ámbito del discurso a través de materiales auténticos en los que el aprendiente pueda ver, aprender y analizar el funcionamiento de dichas unidades.

Si ponemos el foco en los resultados de las preguntas dirigidas a averiguar si los docentes disponen y conocen maneras de poner en marcha propuestas educativas que incluyan audiolibros, podemos concluir que no disponen de información suficiente; a pesar de su interés. Esto nos permite contestar nuestra tercera pregunta de investigación. Como indicábamos anteriormente, la gran mayoría (95\%) de encuestados consideran que sería interesante que los manuales especializados en enseñanza de E/LE tengan el audiolibro en cuenta. Si se atiende a esta propuesta, quizás los resultados obtenidos en las preguntas sobre si alguna vez habían realizado una actividad con audiolibros en la que el 70\% indicó que nunca lo habían hecho, cambien en un futuro. Igualmente, este resultado se relaciona de forma directa con que el 77\% afirma no haber encontrado nunca propuestas educativas que indiquen el uso de audiolibros. A primera vista estas dos últimas afirmaciones pueden parecer negativas, pero contrastan con el gran interés de los docentes por explorar las posibilidades del audiolibro como recurso para la enseñanza de E/LE. De la misma manera, hemos constatado que la gran mayoría no conoce plataformas ya sean generales o especializadas en las que obtener audiolibros, lo cual también supone un escoyo a la hora de tener interés en plantear actividades con audiolibros.

Por último, los comentarios de los participantes en la opción de respuesta abierta al final del cuestionario nos permiten reforzar nuestra premisa inicial sobre el interés de los docentes por los audiolibros. Asimismo, varios 
participantes coinciden en resaltar las posibilidades aprendizaje autónomo que facilitaría el audiolibro. Lo que nos permite afirmar que el audiolibro cumple con las características de una TIC, pues el aprendiente puede realizar las actividades propuestas a un ritmo personalizado. Esta implementación de las TIC permite el cambio hacia un enfoque constructivista centrado en el alumno, que se hace responsable de su propio aprendizaje con la ayuda del profesor. En definitiva, el correcto uso del audiolibro tendría beneficios tanto para el docente como para el aprendiente. Por un lado, el docente tendrá la oportunidad de ampliar su perspectiva de profesionalización al trabajar con una herramienta nueva y con potencial demostrado (Simons, 2010). Por otro lado, el aprendiente tendrá la oportunidad de buscar, seleccionar y analizar la información en diferentes recursos de consulta.

\section{CONCLUSIONES}

Los audiolibros son un recurso con un gran potencial documentado en la enseñanza de lenguas extranjeras, sobre todo en la enseñanza y aprendizaje de la competencia léxica. Como indicábamos al inicio, la presente investigación tenía un objetivo doble: (1) examinar los beneficios del audiolibro como recurso para la enseñanza y aprendizaje de la competencia léxica y (2) situarlo como un recurso de interés y por explorar para aplicarlo en el aula de E/LE como TIC para trabajar dicha competencia. En este sentido, hemos podido constatar a través de nuestro estudio de percepción entre docentes de español el interés que despiertan los audiolibros entre los mismos.

Al investigar el potencial de los audiolibros, centrándonos en sus posibilidades para trabajar la enseñanza y aprendizaje de la competencia léxica en E/ LE, pudimos constatar la falta de estudios centrados en este ámbito concreto; pues la mayoría se centran en la adquisición y aprendizaje de inglés como lengua extranjera. No obstante, a raíz de estudios como el de Chang (2011) podemos afirmar de manera empírica que el audiolibro potencia y acelera la adquisición de nuevo léxico. Por otro lado, los resultados del estudio entre 
docentes de E/LE nos permiten dar respuesta total a dos de las tres preguntas de investigación: los audiolibros son un recurso muy desconocido y menos empleado en el aula de E/LE, pero despierta interés entre los docentes (pregunta n. ${ }^{\circ}$ 1) y, en general, los docentes no disponen o desconocen maneras de poner en marcha propuestas educativas que incluyan el audiolibro (pregunta n. ${ }^{\circ}$ ). Sin embargo, pudimos contestar de manera parcial la pregunta n. ${ }^{\circ}$ 2, pues los resultados de los ítems sobre la utilidad de los audiolibros para trabajar las diferentes competencias señalan que las ideales serían la competencia auditiva y la competencia léxica casi al mismo nivel. Es decir, no se percibe como un recurso óptimo para trabajar únicamente la competencia léxica, sino que sitúa al mismo nivel a la competencia léxica y la comprensión auditiva. Si bien es cierto que ambas están estrechamente relacionadas. Por lo tanto, podemos afirmar que el objeto de nuestro estudio tiene sentido, en tanto que el audiolibro puede ser un recurso con posibilidades para la enseñanza y aprendizaje del léxico en E/LE.

En suma, a pesar de ser conscientes de las limitaciones derivadas de la falta de literatura previa especializada sobre el objeto de estudio, esta investigación evidencia el interés por los audiolibros por parte de los docentes, asî́ como la falta de recursos editoriales especializados y propuestas educativas que faciliten su implementación en el aula de E/LE. Futuras investigaciones podrían enfocarse a realizar estudios empíricos similares a los analizados a lo largo de esta investigación para comprobar si, efectivamente, en el aula de E/LE los audiolibros contribuyen a un mejor y más acelerado aprendizaje de la competencia léxica.

\section{BIBLIOGRAFÍA}

Alcantud-Díaz, M. y Gregori-Signes, C. (2014). Audiobooks: improving fluency and instilling literary skills and Education for development. Tejuelo, 20, 111-125.

Baralo, M. (2005). La competencia léxica en el Marco común europeo de referencia. Carabela, 58, 27-48. SGEL. 
Bergman, O. (1999). Wait for me! Reader control of narration rate in talking books. Reading online.

Blum I., Koskinen, P., Tennant, N.et al (1995). Using audiotaped books to extend classroom literacy instruction into the homes of second language learners. Journal of Reading Behavior, 27, 535-563.

Brown, R., Waring, R., y Donkaewbua, S (2008). Incidental vocabulary acquisition from reading, reading-while-listening, and listening to stories en Reading in a Foreign Language, 20, pp. 136-163.

Chang, A. C.-S (2011). The effect of reading while listening to audiobooks: listening fluency and vocabulary gain en Asian Journal of English Language Teaching, 21,43-64.

Chang, C-S. y Read, J. (2006). The effect of listening support on the listening performance of EFL learners. TESOL Quarterly, 40, 375-397.

Chiriboga, B. (1993). Didáctica del Español como segunda lengua. Abya Yala.

Consejo de Europa (2002). Marco común europeo de referencia para las lenguas: aprendizaje, enseñanza, evaluación. Secretaría General Técnica del MECD y ANAYA.

Cruz Piñol, M. (2015). Léxico y ELE: enseñanza/aprendizaje con tecnologías. Journal of Spanish Language Teaching, 2(2), 165-179. https://doi.org/10.1080/23247797.2015. 1105518

Dirven, R. and Oakeshott-Taylor, J. (1984). Listening Comprehension (Part I). Language Teaching, 17(4): 326-43.

García Giménez, C. (2008). La adquisición del vocabulario en la clase de E/LE. Alicante: Biblioteca Virtual Miguel de Cervantes.

Garrido Rodríguez, M. C. (10-13 de septiembre de 2003). El español coloquial en la enseñanza de ELE. XIV Congreso Internacional de ASELE. Universidad de Burgos, España.

Hernández Mercedes, M. P. (2012). La integración de las TIC en la clase de ELE. Panorama de una (r)evolución. Revista Internacional de Lenguas Extranjeras, 1, 63-99. https:// doi.org/10.17345/rile1.8

Herrera, F. y Conejo, E. (2009). Tareas 2.0: la dimensión digital en el aula de español lengua extranjera. MarcoELE. Revista de didáctica ELE, 9.

Higueras, M. (2017). Logros y retos de la enseñanza del léxico. En F. Herrera (Ed.), Enseñar léxico en el aula de español: el poder de las palabras. (pp. 13-19). Difusión.

Marquès Graells, P. (2000). Los medios didácticos. http://peremarques.pangea.org/medios.htm

Muñoz Coronado, M. (2012). Audiolibros: recursos documentales para el aprendizaje del español como lengua extranjera. Instituto Cervantes de Argel.

Nogueira da Silva, A. M. (2009). La enseñanza de los marcadores del discurso del español en relación con los géneros y secuencias textuales. Revista Nebrija de Lingüistica Aplicada a la Enseñanza de las Lenguas, 9, 1-15. https://doi.org/10.26378/rnlael59156 
Nogueira da Silva, A. M. (2010). Introducción de los marcadores del discurso en los manuales de ELE nivel B2. MarcoELE. Revista de didáctica ELE, 10, 1-26.

Rubery, M. (2016). The Untold Story of the Talking Book. Harvard University Press

Simons, M. (2010). Perspectiva didáctica sobre el uso de las TIC en clase de ELE. MarcoELE. revista de didáctica ELE, 11.

Vallorani, C. M. (2011). La oralidad tecnológica-digital. Estudio pragmático-comunicativo sobre la oralidad en el audiolibro. [Trabajo Fin de Máster, Universidad de Alicante]

Webb, S. y Chang, A. C.-S. (2015). Second language vocabulary learning through extensive reading with audio support: How do frequency and distribution of occurrence affect learning? Language Teaching Research, 19(6), 667-686. https://doi. org $/ 10.1177 / 1362168814559800$

Whittingham, J. et al. (2013). Use of Audiobooks in a School Library and Positive Effects of Struggling Readers' Participation in a Library-Sponsored Audiobook Club. School Library Media Research 16. American Association of School Librarians. 